\title{
BER IMPROVEMENT USING A 2-R REGENERATOR BASED ON AN ASYMMETRIC NONLINEAR OPTICAL LOOP MIRROR
}

\author{
Markus Meissner, ${ }^{1}$ Klaus Sponsel, ${ }^{1}$, Kristian Cvecek, ${ }^{1}$ Andreas Benz, ${ }^{2}$ Stefan \\ Weisser, ${ }^{2}$ Bernhard Schmauss, ${ }^{3}$ and Gerd Leuchs ${ }^{1}$ \\ ${ }^{1}$ Insitute of Optics, Information and Photonics, Max-Planck Research Group, University of \\ Erlangen-Nuernberg, Guenther Scharowskys Str.1, D-91058 Erlangen, Germany ${ }^{2}$ Lucent Tech- \\ nologies, Nurnberg, Germany; ${ }^{3}$ Electrical Engineering Departement, University of Applied \\ Science Regensburg, Germany
}

Abstract: BER improvement of 15 decades is observed using an asymmetric NOLM at a bitrate of $40 \mathrm{Gbit} / \mathrm{s}$ and an input OSNR of $28.7 \mathrm{~dB}$. The BER improvement can be converted into $3.9 \mathrm{~dB}$ of OSNR gain. Both were achieved by optimizing NOLM input power and splitting ratio. This allows for longer spans and thus reduces the over all amount of amplifiers or allows for an increased system reach.

\section{INTRODUCTION}

Signal regeneration is a key element for future data transmission at high bit rates. 3- $\mathrm{R}$ and $2-\mathrm{R}$ regenerators are currently under investigation using all optical techniques. If no retiming is required NOLM based 2-R regenerator a re an interesting alternative to $3-\mathrm{R}$ regenerators, as they are less complex but have high potential for increasing the system reach $[1,2]$. Their set-up uses only a few components and integration in an existing optical transmission system is simple $[1,2]$. We use a $2-\mathrm{R}$ regenerator based on an asymmetric NOLM suggested by Smith and Doran [3]. With the nonlinear power transfer characteristic of the device (see Fig. 2) it is possible to suppress noise and thus to prohibit the formation of new bit errors. The feasibility of such a setup concerning noise reduction and BER improvement was experimentally shown $[4,5]$.

We present for the first time to our knowledge a detailed analysis of the NOLM's impact on the BER. The BER improvement in dependence of the NOLM in- 
put power and splitting ratio is investigated, and the OSNR performance with optimized NOLM parameters is measured.

\section{SIGNAL REGENERATION WITH AN ASYMMETRIC NOLM}

Due to the nonlinear transmission characteristic of the NOLM (e.g. Fig.2) it is possible to reduce amplitude noise imposed on the "1" bits of the signal. In addition the noise on the " 0 " bits is reduced to some degree. By this noise reduction the probability density functions (pdf) of " 1 " and "0" bits are compressed, compensating for the broadening in the preceding EDFA. Therefore the formation of new bit errors due to an overlap of both pdfs is reduced. The NOLM itself is a passive device and thus cannot reverse any already ex isting overlap between the two pdfs, being responsible for the BER. Thus the usage of NOLMs as in-line regenerators has best impact on the signals evolution at the beginning of a transmission-link as done in our experimental setup. Implementation of the NOLM at the end of the link has only little impact on the BER. Furthermore the NOLM should be used at good OSNR around 30dB, as maximum noise reduction is achieved under these conditions [6].

\section{EXPERIMENTAL SETUP}

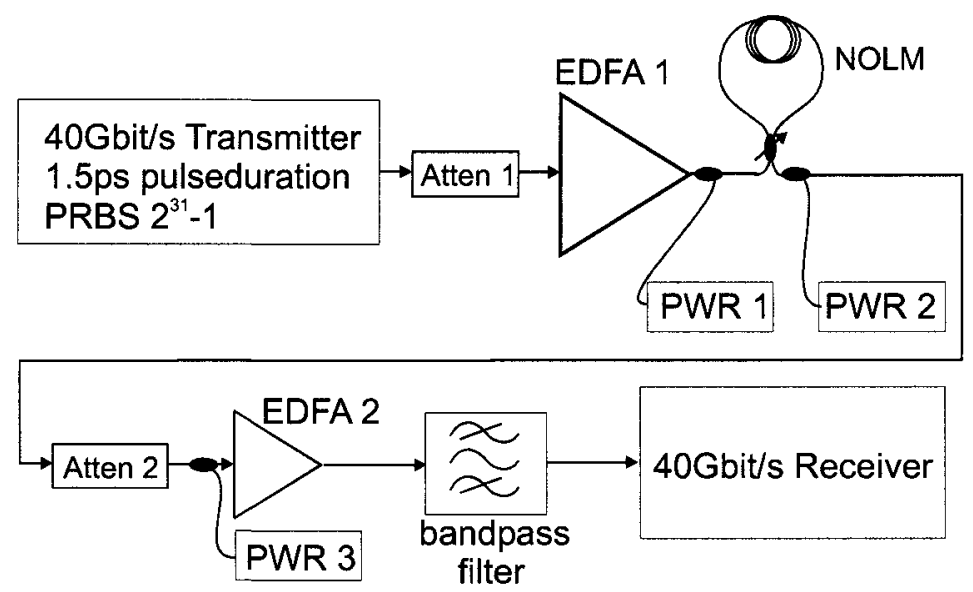

Figure 1. Experimental Setup. The setup emulates a 2 span transmission system in which fiber is emulated by attenuators.

The experimental setup is depicted in Fig. 1. A 1.5ps laser with $10 \mathrm{GHz}$ repetition rate is multiplexed to a $40 \mathrm{~GB} / \mathrm{s}$ pulse train which is modulated with PRBS $2^{31}-1$ data. With attenuator 1 and the following high power EDFA1 the NOLM input OSNR and the NOLM input power are adjusted. The NOLM 
consists of a variable fiber coupler and $1840 \mathrm{~m}$ of NZDSF fiber. After the NOLM the signal is attenuated and amplified again by EDFA2. This emulates a fiber span following the NOLM. The input power of EDFA2 is kept constant to $-24 \mathrm{dBm}$ with attenuator2, thus adding always a constant noise to the NOLM output signal. Then the data signal is band pass filtered and detected.

\section{EXPERIMENTAL RESULTS}

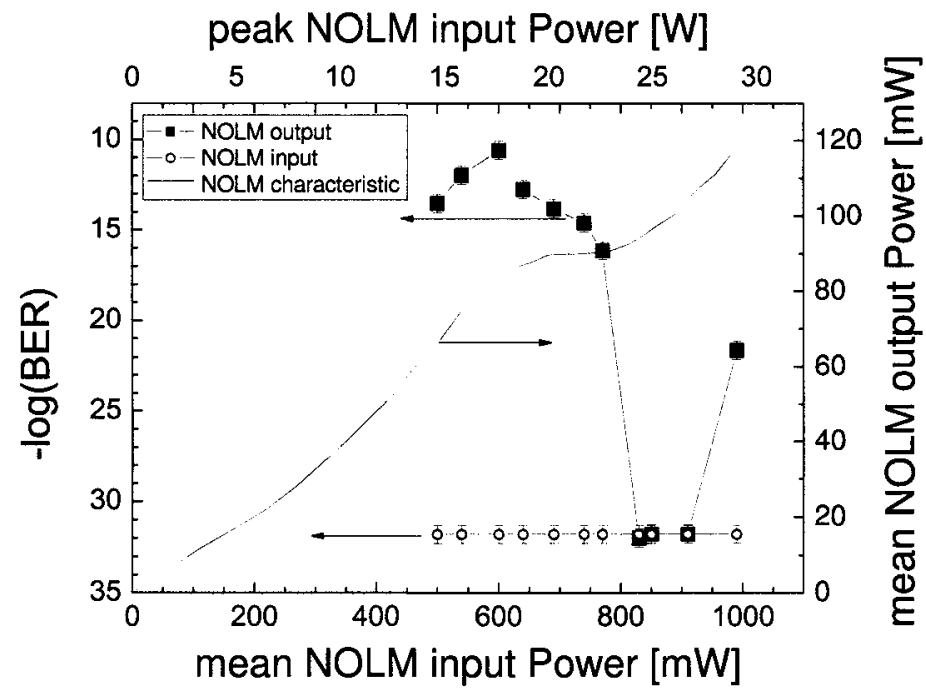

Figure 2. NOLM transfer characteristic and BER measured directly behind the NOLM. BER measured behind EDFA1 is shown, too. The splitting ratio was adjusted to $12: 88$, the OSNR at the output of EDFA1 was $28.7 \mathrm{~dB}$.

We measured the BER versus the decision threshold (V-curves) at different NOLM input powers with the NOLM directly in front of the receiver, excluding attenuator 2 and EDFA 2. From these V-curves the minimum BER was extrapolated. The NOLM input OSNR was set to $28.7 \mathrm{~dB}$, the splitting ratio was 12:88. As shown in Fig.2, the BER taken at the NOLM output, varies with the NOLM input power. For comparison the BER without the NOLM is also shown. It is clearly visible that the NOLM can only degrade the BER in this application, but never improve it. Only at the upper end of the plateau the NOLM induces no BER degradation, and the same level as without the NOLM is reached. Thus, implementing a NOLM at the end of a transmission link, directly in front of the receiver, is shown to be ineffective. Broaden- 


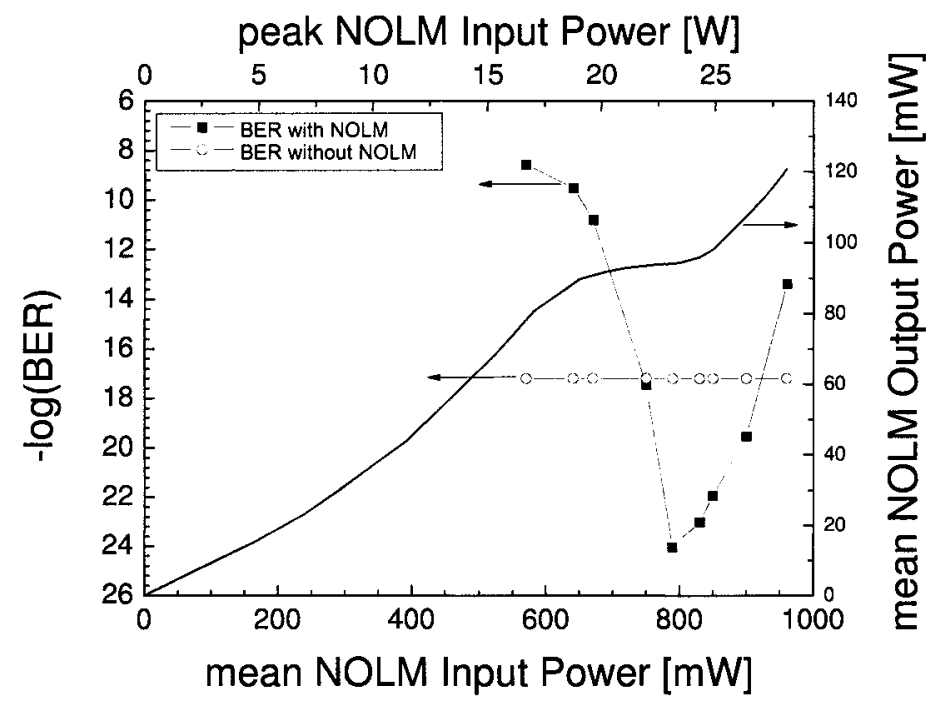

Figure 3. NOLM transfer characteristic, BER with and without NOLM measured at the output port of bandpass filter. The splitting ratio of the NOLM was set to 14:86, the OSNR at the output of EDFAl was set to $28.7 \mathrm{~dB}$

ing the probability density functions of " 1 " and "0"bits with the subsequent EDFA2, by adding noise to the signal after the NOLM, BER improvement can be demonstrated as shown in Fig. 3. The receiver input OSNR was set to $25.7 \mathrm{~dB}$. As expected from the measurement without adding noise, the optimum operation point is the upper end of the plateau region. In these measurements, too, the BER is extrapolated from V-curves. The BER is reduced from $10^{-17}$ to $10^{-25}$. Changing the NOLM input power the BER improvement reduces, and for powers below and above the plateau region even BER degradation may be observed. Varying the splitting ratio the BER can be optimized further, as shown in Fig.4. Best results are achieved for a splitting ratio of 16:84. For more symmetric splitting ratios the BER improvement reduces and for splitting ratios more symmetric than 28:72 the NOLM only degrades the signal. Measuring the OSNR performance of the NOLM leads to Fig. 5. This figure shows the BER with and without NOLM as a function of the output OSNR of EDFA1. Additionally this figure shows the OSNR gain achieved with the NOLM on its right hand side. As expected from noise measurements [6] and Fig.4 adaption of the NOLM splitting ratio on the NOLM input OSNR was necessary to optimize the results. The BER with NOLM was taken at the upper end of the plateau, as shown in Fig.3 for splitting ratios of 12:88 and 


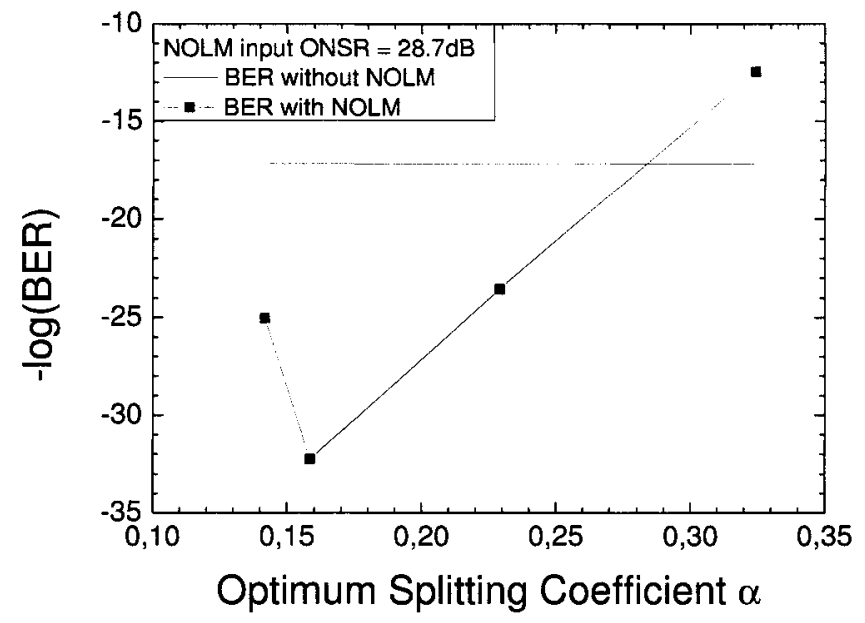

Figure 4. BER taken at the output port of the bandpass filter for different splitting ratios. The BER without NOLM is given for reference. The BER was taken at the upper end of the plateau.

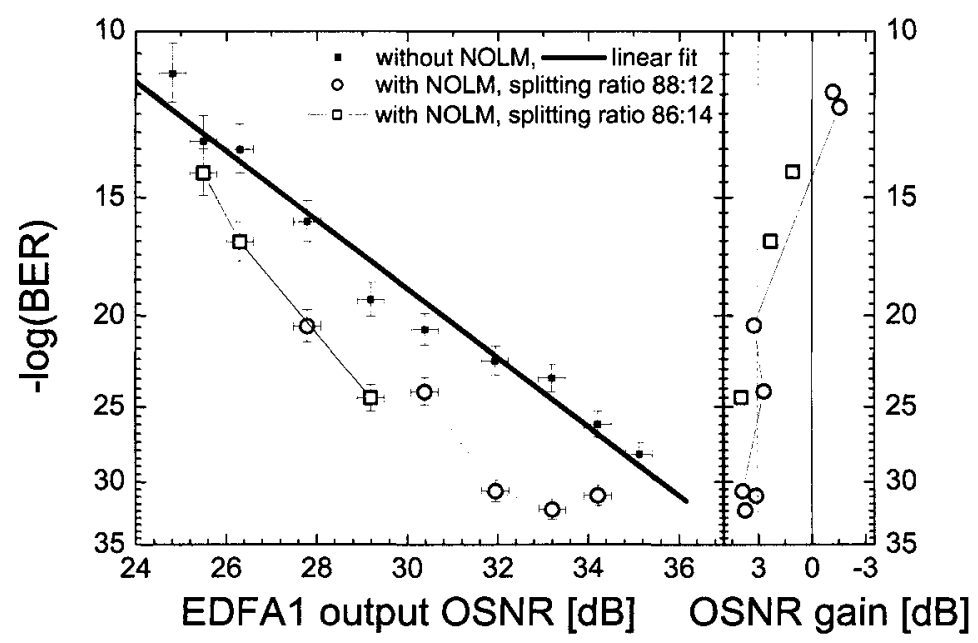

Figure 5. OSNR performance without and with NOLM for different OSNR, taken at the output of EDFA1, and different splitting ratios. 
$14: 86$. For NOLM input ONSRs between $27 \mathrm{~dB}$ and $32 \mathrm{~dB}$, corresponding to BERs between $10^{-21}$ and $10^{-32}$, more than $3 \mathrm{~dB}$ of OSNR gain are achieved. Best OSNR gain of $3.9 \mathrm{~dB}$ is achieved for a BER of $10^{-24}$. Measurement of data for lower OSNR and higher BER could not be performed due to a lack of power, provided by EDFA1. At low OSNR a large part of EDFA1's pump power generates ASE noise, thus reducing the power available for signal amplification. Currently the power needed to operate the NOLM is very high. But with the development of modern photonic crystal fibers, fibers will be available which have a high nonlinearity and thus can reduce the power requirement to a few $\mathrm{mW}$.

\section{CONCLUSIONS}

BER improvement by a 2-R regenerator based on an asymmetric NOLM has been studied. 15 decades of BER improvement at a NOLM input OSNR of $28.7 \mathrm{~dB}$ were demonstrated after optimizing the splitting ratio to $16: 84$. For optimum operation the NOLM input power has to reach the upper end of the plateau. More than 3dB gain of OSNR were observed for NOLM input OSNR between $27 \mathrm{~dB}$ and $32 \mathrm{~dB}$ by implementing the NOLM into a transmission link. A maximum OSNR gain of $3.9 \mathrm{~dB}$ was observed for NOLM splitting ratio of $86: 14$ and a BER of $10^{-24}$. Thus by using the NOLM as an in-line regenerator the system reach can be more than doubled. Alternatively the length of a span can be elongated by up to $19 \mathrm{~km}$, as up to $3.9 \mathrm{~dB}$ of extra attenuation can be tolerated. This in turn can be used to reduce the amount of amplifiers needed for a transmission system. The amount of amplifiers used for example in the FLAG-Atlantic-1 cable can be reduced by $31 \%$ when using NOLMs.

\section{ACKNOWLEDGMENTS}

We gratefully acknowledge the financial support of the Deutsche Forschungsgemeinschaft (DFG) and the Bayrische Staatsregierung.

\section{REFERENCES}

[1] Z. Huang, A. Gray, Y. W. A. Lee, I. Khrushchev and Ian Bennion: "40GB/S Transmission over $4000 \mathrm{Km}$ of standard fibre using in-line nonlinear optical loop mirror", Proc. ECOC, Rimini, Italy, Vol. 1, Mo4.6.3, 2003

[2] F. Seguineau, B. Lavigne, D. Rouvillain, P. Brindel, L. Pierre, and O. Leclerc: "Experimental demonstration of simple NOLM based 2-R regenerator for $42.66 \mathrm{GBit} / \mathrm{s}$ WDM long-haul transmissions", Proc. OFC, Los Angeles, USA, WN4, 2003

[3] N.J. Smith and N.J. Doran:"Picosecond soliton transmission using concatenated nonlinear optical loop-mirror intensity filters" J. Opt. Soc. Am. B, Vol. 12, pp. 1117-1125, 1995.

[4] M. Meissner, M. Roesch, B. Schmauss, G. Leuchs:“12dB of noise reduction by a NOLM based 2-R-regenerator",Photonics Technology Letters, Vol. 15, pp. 1297-1299, 2003 
[5] R. Ludwig, A. Sizmann, U. Feiste, C. Schubert, M. Kroh, C.M. Weinert and H.G. Weber :"Experimental Verification of Noise Squeezing by an Optical Intensity Filter in HighSpeed Transmission", Proc. ECOC, Amsterdam, Netherlands, 2001

[6] M. Meissner, M. Roesch, B. Schmauss and G. Leuchs: "Noise reduction performance of a NOLM based 2-R-regenerator in dependence on the OSNR", Proc. ECOC, Rimini, Italy, Vol. 3, P3.08, 2003 